\title{
Molecular Phylogenetics of Aedes japonicus, a Disease Vector That Recently Invaded Western Europe, North America, and the Hawaiian Islands
}

\author{
EMILIE C. CAMERON, ${ }^{1}$ RICHARD C. WILKERSON,${ }^{2}$ MOTOYOSHI MOGI ${ }^{3}$ ICHIRO MIYAGI ${ }^{4}$ \\ TAKAKO TOMA, ${ }^{4}$ HEUNG-CHUL KIM, ${ }^{5}$ AND DINA M. FONSECA ${ }^{1,6}$
}

J. Med. Entomol. 47(4): 527-535 (2010); DOI: 10.1603/ME09259

\begin{abstract}
We used two mitochondrial loci (nicotinamide adenine dinucleotide dehydrogenase subunit 4 and cytochrome oxidase II) and a nuclear locus (28S-D2 spacer) for a total of $1337 \mathrm{bp}$ to evaluate the relationships among the four subspecies of Aedes (Finlaya) japonicus Theobald. Ae. $j$. japonicus was recently introduced into the United States and has been expanding rapidly. We also included in our analysis a morphologically very closely related species, Aedes (Finlaya) koreicus Edwards, as well as three more distantly related species: Aedes (Finlaya) togoi Theobald, Aedes (Finlaya) hatorii Yamada, and Aedes (Aedimorphus) vexans Meigen. We found that the four subspecies in the Ae.japonicus complex are genetically quite distinct but seem to form a monophyletic group that surprisingly also includes Ae. koreicus, suggesting the need for a taxonomic reconsideration of the group. We also found that the two southern subspecies are more closely related to each other than to any of the remaining subspecies or to Ae. koreicus and may indicate an ancient north-south split of the lineage. Considering the overlap between Ae.j. japonicus and Ae. koreicus, but the stronger association between Ae. koreicus and humans, we are surprised it also has not expanded from its original range. As a proactive reaction to this possibility, we designed and tested a DNA-based rapid assay to differentiate Ae. koreicus from some of the species with which it may be confused in the United States. These Aedes are putative vectors of several important viral encephalitides.
\end{abstract}

KEY WORDS cytochrome oxidase II, nicotinamide adenine dinucleotide dehydrogenase subunit 4, ITS-D2 spacer, Aedes koreicus, Ochlerotatus japonicus

The genus Aedes (Diptera: Culicidae) includes the principal vectors of yellow fever, dengue, and aperiodic lymphatic filariasis (Foster and Walker 2002). This is a diverse taxon currently containing 921 species (www.mosquitocatalog.org; but also see http:// mosquito-taxonomic-inventory.info/valid-species-list and Reinert 2000, Reinert et al. 2004, 2006, 2008), which is in need of a detailed examination (Black 2004, Savage and Strickman 2004, Tabachnick 2005). A few

The opinions or assertions contained herein are the private views of the author, and are not to be construed as official, or as reflecting true views of the Department of the Army or the Department of Defense.

${ }^{1}$ Center for Vector Biology, Rutgers University, 180 Jones Ave., New Brunswick, NJ 08901.

${ }^{2}$ Walter Reed Biosystematics Unit, Division of Entomology (WRBU), Walter Reed Army Institute of Research, 503 Robert Grant Ave., Silver Spring, MD 20910-7500.

${ }^{3}$ Department of Microbiology, Saga Medical School, Nabeshima 5-1-1, Saga 849-8501, Japan.

${ }^{4}$ Laboratory of Medical Zoology, School of Health Sciences, Faculty of Medicine, University of the Ryukyus, Nishihara, Okinawa 903-0215, Japan.

5 th Medical Detachment, 168th Multifunctional Medical Battalion, 65th Medical Brigade, U.S. Army, APO AP 96205-5247, Republic of Korea.

${ }^{6}$ Corresponding author, e-mail: dinafons@rci.rutgers.edu. species in this group of mosquitoes have historically expanded their global distribution in association with humans (Tabachnick and Powell 1979). Many Aedes are container breeders that take advantage of water accumulated in human refuse and/or have an egg stage that can withstand dehydration. In particular, members of this genus seem to be especially able to exploit the current extensive worldwide trade in used tires and similar containers (Lounibos 2002). The introduction of the African Aedes (Stegomyia) aegypti to the New World heralded epidemics of yellow fever as well as dengue fever (Tabachnick and Powell 1979), and the introduction of Ae. (Stg.) albopictus exacerbated the scourge of dengue fever in Central and South America, if not in North America (Gratz 2004, Paupy et al. 2009), and has become the primary vector of chikungunya fever virus, leading to recent epidemics in Asia and Europe (de Lamballerie et al. 2008).

Aedes (Finlaya) japonicus japonicus Theobald (Diptera: Culicidae) was first collected outside its native range of northeast Asia (Tanaka et al. 1979) in 1998 when it was collected in the United States (Peyton et al. 1999, Andreadis et al. 2001). Although it is unclear when it was first introduced to the United States, the fact that extensive collections aimed at 
detecting the presence of Ae. albopictus (Moore et al. 1990) in 1992 failed to uncover Ae. j. japonicus argues that it must have been introduced since 1992 (Andreadis et al. 2001). Ae. japonicus has expanded in North America from three states in 1998 (Connecticut, New York, and New Jersey) to a current total of 31 (Alabama, Connecticut, Delaware, Georgia, Hawaii, Iowa, Illinois, Indiana, Kentucky, Massachusetts, Maryland, Maine, Michigan, Minnesota, Missouri, North Carolina, New Hampshire, New Jersey, New York, Ohio, Oregon, Pennsylvania, Rhode Island, South Carolina, South Dakota, Tennessee, Virginia, Vermont, Washington, Wisconsin, and West Virginia) as well Quebec, Canada (Larish and Savage 2005, Widdel et al. 2005, Saenz et al. 2006, Bevins 2007, Morris et al. 2007, Hughes et al. 2008,Neitzel et al. 2009). The species is very common in northeastern states, e.g., Pennsylvania, Connecticut, and New York (Andreadis et al. 2001, Falco et al. 2002); and although it is inexorably expanding south and into the Midwest, its presence there is still very localized (Joy 2004, Roppo et al. 2004, Qualls and Mullen 2006, Bevins 2007). Breeding populations also were found in France in 2000 (Schaffner et al. 2003) and in Belgium in 2002 (Versteirt et al. 2009).

Ae. (Fin.) japonicus is currently composed of four geographically distinct subspecies: Ae. j. japonicus, Ae. j. shintienensis Tsai \& Lien, Ae. j. yaeyamensis Tanaka, Mizusawa \& Saugstad, and Ae. j. amamiensis Tanaka, Mizusawa \& Saugstad. The subspecies Ae. j. japonicus is very common in Palearctic Japan and shows little morphological variation there. Ae. j. japonicus also is found in Korea and throughout China (Lu et al. 1997), although it is not clear whether this is the only subspecies found there. Common on Yaeyama Gunto, the southern most islands of the Ryukyu Archipelago, Ae. $j$. yaeyamensis is quite distinct from the other subspecies, although the diagnostic hind femur scale pattern clearly overlaps Ae. (Fin.) koreicus, a closely related species found in Korea and China, making misidentification likely (Fig. 1). Tanaka et al. (1976) attributes the morphological differences between Ae. $j$. yaeyamensis and the other subspecies to it having become isolated earlier in the evolution of the complex. Ae. $j$. shintienensis, which occurs in Taiwan, closely resembles its northern neighbor Ae. $j$. yaeyamensis in the aedeagus, implying a possible common ancestor. Aedes j. amamiensis is found in Amami Ôshima, the northernmost Islands of the Ryukyu Archipelago, and although not as clearly defined morphologically as Ae. $j$. yaeyamensis it is still considered a distinct subspecies. Interestingly, only two specimens collected in Okinawa, in central Ryuku Archipelago, have been identified as Ae. japonicus. These specimens were morphologically similar to Ae.j. amamiensis (Toma and Miyagi 1981, 1986).

Close examination of the morphological evidence indicates there are insufficient diagnostic traits to reliably separate the subspecies in the Ae. japonicus complex from each other and from Ae. koreicus. As adults, the primary character separating them is the degree of development of the sub-basal dark band

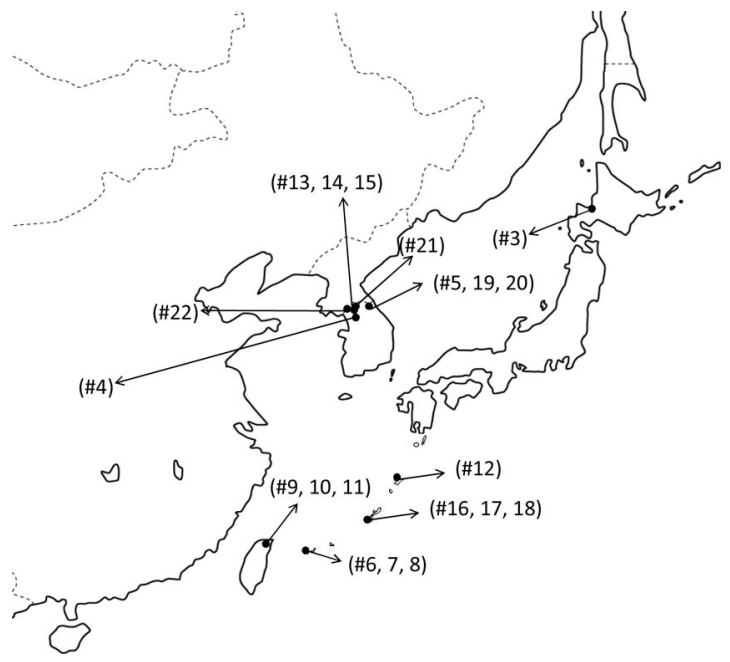

Fig. 1. Sampling locations in East Asia. Numbers refer to those in Table 1.

(Fig. 1). Additional morphological characters identifying specimens as Ae. koreicus have a generous amount of overlap with Ae. japonicus and the overlap in characters could lead to possible misidentification of adult Ae. japonicus (especially yaeyamensis) as Ae. koreicus (Tanaka et al. 1979).

Substantial similarity of morphological characters, in particular the male genitalia, suggests that $\mathrm{Ae} . \mathrm{ko}$ reicus may be more closely related to Ae.japonicus than suggested previously. The phylogenetic structure of the genus Aedes, including subgenus Finlaya, has been thus far largely without molecular data. We therefore sought to test for concurrence of molecular and morphological characters.

Our objectives were to examine the relationships between members of the Ae. japonicus complex both to understand patterns of evolution within the genus Aedes as well as to develop better diagnostic markers. Ae. j. japonicus and Ae. koreicus have been shown to be efficient laboratory and field vectors of several encephalitides, including West Nile virus and Japanese encephalitis (Tanaka et al. 1979; Takashima and Rosen 1989; Turell et al. 2001; Sardelis et al. 2002a,b; Kutz et al. 2003, Sardelis et al. 2003), which makes understanding and attempting to curb their expansion across the World critical.

\section{Materials and Methods}

For phylogenetic analysis, we sequenced three gene regions in 20 specimens collected from East Asia and two from the United States. We included specimens from the four subspecies of Ae. japonicus as well as specimens of Ae. koreicus, Aedes (Finlaya) togoi Theobald, Aedes (Finlaya) hatorii Yamada, and Aedes (Aedimorphus) vexans Meigen, with the latter three species as outgroups (Fig. 2; Table 1). Specimens were collected, identified based on morphology, and stored either dry or in ethanol, before DNA extraction. Total 


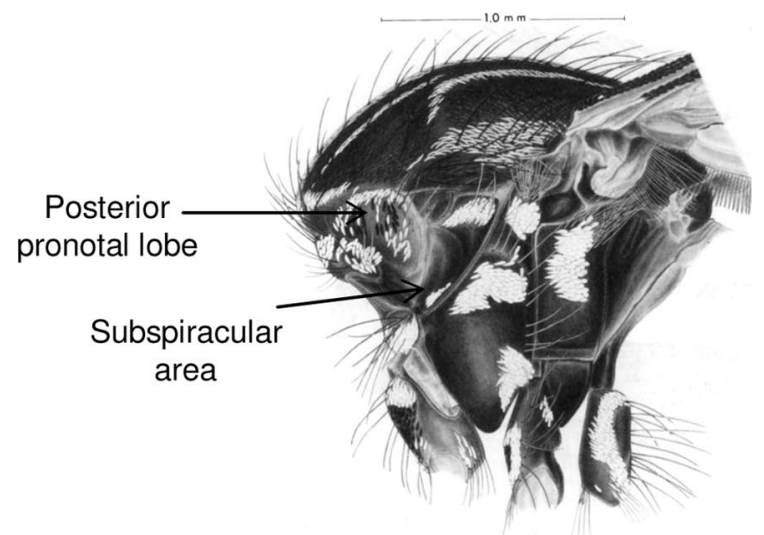

Ae. (Fin.) koreicus

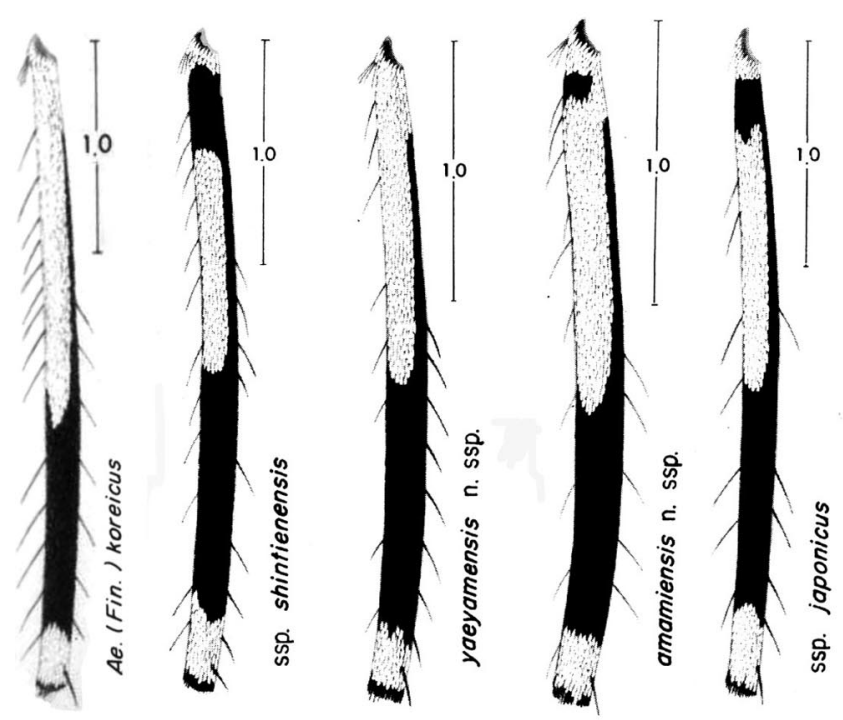

Fig. 2. Comparison of the thorax and hind femora of Ae. koreicus and Ae.japonicus subspecies. In Ae. koreicus, the posterior pronotal lobe has dark scales in $89.2 \%$ of specimens, whereas in Ae. j japonicus $7.3 \%$ have them. The subspiracular area has a distinct patch of pale scales in $87.8 \%$ of Ae. koreicus, whereas $93 \%$ of Ae.j japonicus lack any scales in this area (Tanaka et al. 1979). In addition, larvae of Ae. koreicus always lack detached simple pectin teeth, a character that is very rare in Ae. japonicus s.l. Pictures modified from Tanaka et al. (1979) (drawings by S. Shibata).

genomic DNA was extracted from individual whole mosquitoes by using a phenol chloroform extraction method as described in Fonseca et al. (2000).

DNA was amplified from each specimen at two mitochondrial loci, nicotinamide adenine dinucleotide dehydrogenase subunit 4 (ND4) and cytochrome oxidase II (COII), and one nuclear locus, $28 \mathrm{~S}$ ribosomal subunit spacer 2 (D2). For ND4, we used the primers N4J-8502D (5'-CGTAGGAGGAGCAGCTATATT-3') and N4N-8944D (5'-AAGGCTCATGTTGAAGCTCC- $3^{\prime}$ ) as described in Fonseca et al. (2001). Partial COII sequences were obtained using the primers Pierre (5'-AGTTCATCTCCTTTAATAGAACA-3') and Barbara (5'-TGGTCAATGTTCAGAAATTTGTGG-3') modified from Simon et al. (1994). The D2 variable expansion region of $28 \mathrm{~S}$ rRNA was amplified using primers D2 F (5'-AGTCGTGTTGCTTGATAGTG- $3^{\prime}$ ) and D2R (5'-CTTGGTCCGTGTTTCAAGAG-3') from Sallum et al. (2002). Each reaction was carried out in a $50-\mu \mathrm{l}$ volume, with final concentrations of $1 \times$ polymerase chain reaction (PCR) buffer, $300 \mathrm{nM}$ of each primer, $250 \mathrm{mM}$ of each dNTP, $2 \mathrm{mM} \mathrm{MgCl}$ ( $2.5 \mathrm{mM}$ for COII), $1 \mathrm{mg} / \mathrm{ml} \mathrm{BSA}$ $(0.4 \mathrm{mg} / \mathrm{ml}$ for D2), $0.05 \mathrm{U}$ of Taq Gold polymerase (Applied Biosystems, Foster City, CA) and 5 ng of DNA. The PCR amplification consisted of a 10-min denaturation at $96^{\circ} \mathrm{C}, 40$ cycles of $40 \mathrm{~s}$ at $94^{\circ} \mathrm{C}, 40 \mathrm{~s}$ at $55^{\circ} \mathrm{C}\left(50^{\circ} \mathrm{C}\right.$ for COII and $48^{\circ} \mathrm{C}$ for D2), and 1 min at $72^{\circ} \mathrm{C}$ ending with a final extension step of $10 \mathrm{~min}$ at $72^{\circ} \mathrm{C}$.

Cycle sequencing was performed using Big Dye 3.1 chemistry (Applied Biosystems) after cleaning the 
Table 1. Sample ID, collection location and source of specimens used in the phylogenetic analyses (all specimens were collected between 1997 and 2000 )

\begin{tabular}{|c|c|c|c|c|c|}
\hline & ID & Species & Country & Region & Received from \\
\hline $1^{a}$ & Aej 3 & Ae.j.japonicus & USA & Long Island, NY & $\begin{array}{l}\text { S. Campbell (Suffolk Vector Control, } \\
\text { Yaphank, NY) }\end{array}$ \\
\hline 2 & Aej 67 & Ae.j.japonicus & USA & Franklin Co, PA & $\begin{array}{l}\text { B. Pagac (Army Center for Health } \\
\text { Promotion and Prevention Medicine- } \\
\text { North, Fort Meade, MD) }\end{array}$ \\
\hline 3 & Aej 33 & Ae.j.japonicus & Japan & Sapporo & $\begin{array}{l}\text { I. Takashima (Hokkaido University, } \\
\text { Sapporo, Japan) }\end{array}$ \\
\hline 4 & Aej 154 & Ae.j.japonicus & Korea & Gyeonggi-Forest Station & M. Mogi (Saga Medical School, Saga, Japan) \\
\hline 5 & Aej 193 & Ae.j.japonicus & Korea & Gangwon Do & H.-C. Kim (U.S. Army, Republic of Korea) \\
\hline 6 & Aejya 79 & Ae.j. yayaemensis & Japan & Iriomotejima, Yaeyama Islands & $\begin{array}{l}\text { I. Miyagi (University of the Ryukyus, } \\
\text { Okinawa, Japan) }\end{array}$ \\
\hline 7 & Aejya 80 & Ae.j. yayaemensis & Japan & Iriomotejima, Yaeyama Islands & I. Miyagi \\
\hline 8 & Aejya 81 & Ae. $j$. yayaemensis & Japan & Iriomotejima, Yaeyama Islands & I. Miyagi \\
\hline 9 & Aejsh 138 & Ae.j. shintienensis & Taiwan & Tsouko, Sanhain twp, Taipe County & $\begin{array}{l}\text { J. C. Lien (National Taiwan University, } \\
\text { Taipei, Taiwan) }\end{array}$ \\
\hline 10 & Aejsh 139 & Ae.j. shintienensis & Taiwan & Tsouko, Sanhain twp, Taipe County & J. C. Lien \\
\hline 11 & Aejsh 140 & Ae. $j$. shintienensis & Taiwan & Tsouko, Sanhain twp, Taipe County & J. C. Lien \\
\hline 12 & Aejam 386 & Ae.j. amamiensis & Japan & $\begin{array}{l}\text { Amami \& Omacr;shima, Ryukyu } \\
\text { Islands }\end{array}$ & M. Mogi \\
\hline 13 & Aek 3 & Ae. koreicus & Korea & CampCasey, Gyeonggi Do & $\begin{array}{l}\text { D. Claborn (Uniformed Services University } \\
\text { of Health Sciences, Bethesda, MD) }\end{array}$ \\
\hline 14 & Aek 4 & Ae. koreicus & Korea & CampCasey, Gyeonggi Do & D. Claborn \\
\hline 15 & Aek 5 & Ae. koreicus & Korea & CampCasey, Gyeonggi Do & D. Claborn \\
\hline 16 & Aet 1 & Ae. togoi & Japan & Okinawa & I. Miyagi \\
\hline 17 & Aet 2 & Ae. togoi & Japan & Okinawa & I. Miyagi \\
\hline 18 & Aet 5 & Ae. togoi & Japan & Okinawa & I. Miyagi \\
\hline 19 & Aeho 1 & Ae. hatorii & Korea & Gangwon Do & H.-C. Kim \\
\hline 20 & Aeho 2 & Ae. hatorii & Korea & Gangwon Do & H.-C. Kim \\
\hline 21 & Aev 1 & Ae. vexans & Korea & Pochon, Gyeonggi Do & H.-C. Kim \\
\hline 22 & Aev 2 & Ae. vexans & Korea & Daeseong-dong, Gyeonggi Do & H.-C. Kim \\
\hline
\end{tabular}

${ }^{a}$ Numbers correspond to sample numbers indicated on the map in Fig. 2.

PCR products using the QIAquick PCR purification kit (QIAGEN, Valencia, CA). Cycle sequencing products were cleaned with Sephadex columns (Princeton Separations, Adelphia, NJ) before being run on an ABI3700.

Sequences were edited using Sequencher 4.2.2 (GeneCodes, Ann Arbor, MI) and aligned using CLUSTALW (Thompson et al. 1994) with manual adjustments made in BioEdit 7.0.9 (Hall 1999). For each locus, the model of nucleotide evolution that best fit the data were determined in MrModeltest as implemented in MrMTgui 1.0 (Posada and Crandall 1998, Nylander 2004, Nuin 2006), using the Akaike Information Criterion (AIC) and supported by the hierarchical likelihood ratio test. The model selected for the ND4 data set was a HKY + G model, for COII it was a GTR $+\mathrm{I}+\mathrm{G}$ model, and for D2 a GTR $+\mathrm{I}$ model. Bayesian phylogenetic analysis was conducted using these models in MrBayes 3.1.2 (Ronquist and Huelsenbeck 2003) for each locus separately and for a combined partitioned data set. One cold and three heated chains (temperature $=0.075$ ) were run for 5 million generations in two independent MCMC searches. Trees were sampled every 1,000 generations with the first 500 sampled trees discarded as burn-in. Posterior probabilities for each node were obtained with $50 \%$ majority consensus. For comparison, maximum parsimony (MP) trees were also constructed for each locus and the combined data set. MP analysis was performed in PAUP* 4.0b10 (Swofford 2000) by using a heuristic search with tree bisection reconnection (TBR) branch swapping. Branch support was estimated using 10,000 replicate bootstrap resamplings. For D2 there were very small differences between some taxa, thus an alternative method to search the treespace was used as described by Edgecombe et al. (2000). This method performed 1,000 random addition sequence replicates sampling three trees per iteration. Bootstrapping was performed in a similar manner. Shortest trees and 50\% majority consensus trees were observed in FigTree 1.2.2 (Rambaut 2009). To examine the robustness of the nodes of the combined parsimony tree, we calculated the Bremer support in TREEROT version 3 (Sorenson and Franzosa 2007). The percentage difference within and between species was calculated by counting the number of nucleotide differences, in BioEdit 7.0.9.0 (Hall 1999), over the total number of bases sequenced.

\section{Results}

Sequences were obtained for all 22 specimens at three loci: ND4 (348 bp; GenBank accessions GU229919-GU229934), COII (509 bp; GU229893GU229908), and D2 (480 bp; GU229909-GU229918). Under the MP analysis, the combined tree had 243 parsimony-informative characters (ND4, 73; COII, 118 ; and D2, 50), resulting in two trees $(14,4, \mathrm{NA}$ due to method used) of length 444 (146, 226, and 68). Following Bayesian analysis the standard deviation of 
a)

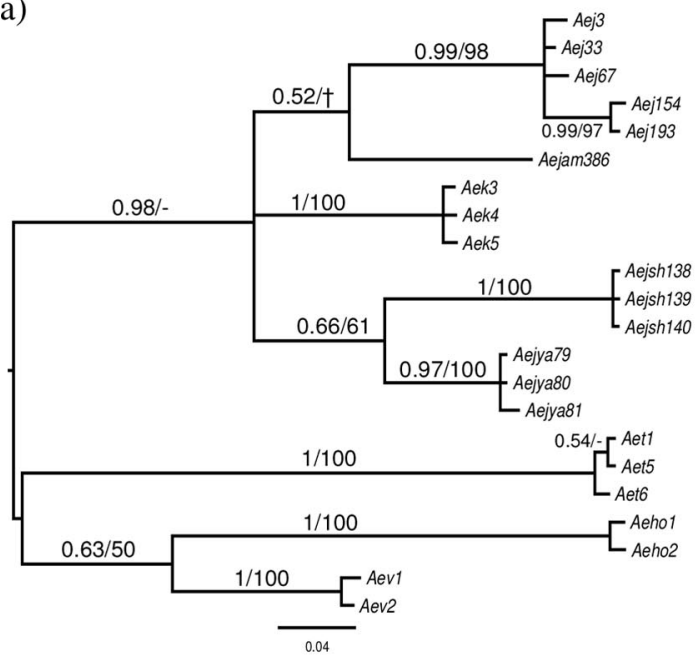

c)

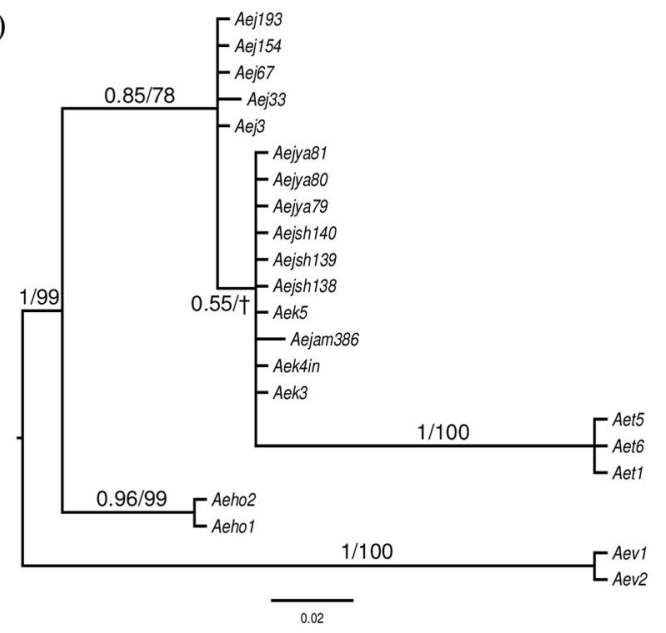

b)

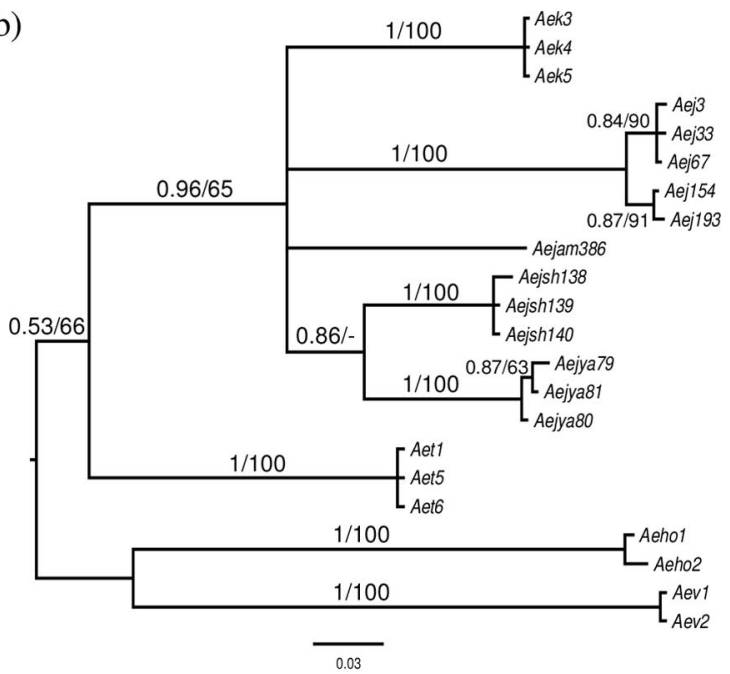

d)

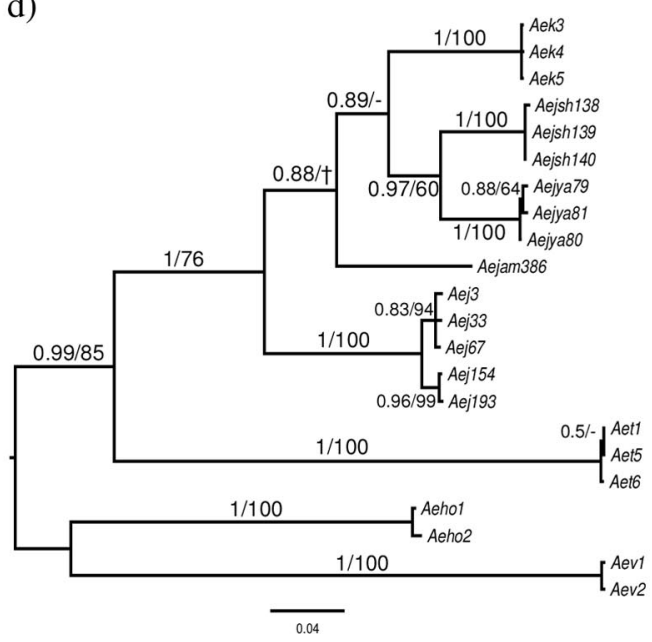

Fig. 3. Bayesian 50\% majority rule consensus trees for ND4 (a), COII (b), D2 sequence data (c), and the combined data set (d). For each node, the Bayesian posterior probability and parsimony bootstrap support are shown (- indicates $<50 \%$ support; $\uparrow$ indicates the node was not recovered by that method).

the split frequencies was $<0.005$ and the average value for the assessed potential scale reduction factors was 1.00 for all loci, indicating that convergence was met in all cases.

In the combined tree Ae. japonicus s.l. and Ae. koreicus form a monophyletic group with strong posterior probability and moderate bootstrap support (Fig. 3d). The Bayesian analysis fully resolved the relationships between the taxa, grouping the two southern subspecies, Ae. j. shintienensis and Ae. j. yaeyamensis, and joining them with Ae. koreicus and Ae. j. amamiensis as a sister taxa to Ae. j. japonicus. The MP analysis, however, did not resolve the relationships between the subspecies. This was further highlighted by low and negative Bremer support values within this clade indicating conflicting signals from the three genes. Overall the Bremer support values were higher for the two mitochondrial genes (ND4, 69.6; COII, 91.5; and D2, 44.9).
Individually the gene trees do not show such a clear pattern. For the mitochondrial markers, ND4 and COII, Bayesian and MP analyses gave very similar results (Fig. 3a and b), with a few nodes not recovered using MP. A monophyletic grouping of Ae. japonicus s.l. and Ae. koreicus was recovered, but within this grouping the relationships between the subspecies are unresolved with the exception of Ae. $j$. shintienensis and Ae. j. yaeyamensis, which cluster together. For ND4, the MP tree has low bootstrap support for placing Ae. togoi outside the japonicus-koreicus clade, thus leaving the relationships between all the taxa unclear. In all cases, multiple individuals from within a taxa grouped together. In contrast, the D2 phylogeny failed to recover the subspecies (Fig. 3c) due to very small differences between these taxa (Table 2). The Bayesian and MP trees disagreed in the placement of $A e$. togoi, which under a Bayesian framework, was placed in the japonicus-koreicus clade, although at a large 
Table 2. Percentage nucleotide difference between the taxa based on the number of nucleotide differences over the total length of the sequences averaged over specimens ${ }^{a}$

\begin{tabular}{lrrrccccc}
\hline \hline & Aev & Aeho & Aet & Aek & Aej & Aejam & Aejsh & Aejya \\
\hline Aev & $\mathbf{0 . 1}$ & 7.9 & 10.0 & 7.9 & 7.9 & 8.1 & 8.1 & 8.1 \\
Aeho & 9.7 & $\mathbf{0 . 4}$ & 8.8 & 4.4 & 4.4 & 4.6 & 4.6 & 4.6 \\
Aet & 9.7 & 10.3 & $\mathbf{0 . 0}$ & 6.5 & 6.7 & 6.9 & 5.3 & 5.3 \\
Aek & 10.8 & 11.2 & 10.3 & $\mathbf{0 . 2}$ & 1.2 & 0.8 & 0.5 & 0.5 \\
Aej & 10.7 & 11.4 & 9.5 & 9.2 & $\mathbf{0 . 9}$ & 1.1 & 0.7 & 0.7 \\
Aejam & 9.6 & 10.6 & 9.0 & 8.3 & 8.6 & - & 0.8 & 0.8 \\
Aejsh & 9.8 & 10.7 & 10.3 & 8.0 & 8.9 & 7.9 & $\mathbf{0 . 1}$ & 0.0 \\
Aejya & 9.6 & 11.4 & 10.5 & 8.7 & 8.5 & 7.7 & 6.3 & $\mathbf{0 . 3}$ \\
\hline
\end{tabular}

${ }^{a}$ The mitochondrial genes are combined in the bottom triangle, and the nuclear D2 marker is in the top triangle. Within-species variation is in bold on the diagonal calculated over all three markers. The species abbreviations are the same as in Table 1.

distance from these species. The MP analysis placed Ae. togoi outside the japonicus-koreicus clade with bootstrap support for the clade of $68 \%$. In addition, MP recovered an Ae. $j$. japonicus clade with bootstrap support of $73 \%$, leaving the remaining subspecies and Ae. korecicus unresolved.

The minimum percent difference among subspecies in the Ae.japonicus complex at the mitochondrial loci was $6.3 \%$, comparable to that between any of the subspecies and Ae. koreicus (Table 2). Indeed, even comparisons with the three outgroup species did not yield considerably higher percent differences. For the D2 locus, comparisons between members of the $A e$. japonicus complex and the outgroup species, Ae. togoi, Ae. hatorii, and Ae. vexans, produced high percent differences; but again, Ae. koreicus yielded similar values to those obtained in comparisons among subspecies.

Ae. koreicus Assay. To aid in the identification of Ae. koreicus, we designed a molecular assay based on the ND4 sequences obtained in this study. At site 151 of our ND4 sequence alignment (corresponding to site 181 in GenBank sequences DQ470164-470154), the three Ae. koreicus have a T, whereas the five Ae. $j$. japonicus have a $\mathrm{G}$ and the other species have an $\mathrm{A}$; thus, this site was chosen to design a primer unique to Ae. koreicus (ND4korF 5'-CCCCATTTAACCCCCAATAT- $\left.3^{\prime}\right)$. The identification assay can be performed as a multiplex PCR with the primers N4J8502D (F) and N4N-8944D(R), ND4korF, with the conditions used to amplify ND4 (described above) adjusting the final concentration of the two forward primers to $0.2 \mu \mathrm{M}$. The assay gives a single band of $465 \mathrm{bp}$ in Ae. $j$. japonicus (13 samples tested), Ae. $j$. shintienensis (3), Ae. j. yaeyamensis (3), Ae. j. amamiensis (1), Ae. togoi (2), Ae. hatorii (2), and Ae.vexans (1) as well as Ae. atropalpus (1) and Ae.triseriatus (3). We tested 26 specimens of A . koreicus, both field collected and museum specimens (Smithsonian Institution, Washington, DC) from the southern Korea Peninsula. All displayed a band of $283 \mathrm{bp}$ as well as a the expected 465-bp band. We also compared the ND4 haplotypes found in Ae. koreicus with all the haplotypes recovered from Ae.j.japonicus across the World (26 haplotypes in $>300$ specimens, Fonseca et al. 2001,
Fonseca et al. 2010) and found that this SNP was consistent. Thus, this assay should provide a useful tool for the early identification of Ae. koreicus.

\section{Discussion}

The primary conclusion of this study is that the four subspecies in the Ae.japonicus complex are genetically quite distinct, averaging $\approx 8 \%$ nucleotide differences at the two mitochondrial loci. Furthermore, they seem to form a monophyletic group that surprisingly also includes Ae. koreicus.

We chose the genetic regions for this analysis based on previous knowledge of their polymorphism (Simon et al. 1994, Fonseca et al. 2001). ND4 was used successfully in population level analysis of Ae. japonicus both from their natural range in Japan and introduced populations in the United States (Fonseca et al. 2001, 2010). Among the 29 Japanese specimens of Ae. $j$. japonicus collected from Nagasaki and Saga (Kyushu), Hiroshima and Tokyo (Honshu), and Sapporo and Chitose (Hokkaido). Fonseca et al. (2001) identified only 11 polymorphisms at the ND4 locus. An analysis that includes all these specimens results in an ND4tree with the same topology as the tree presented here (data not shown). Preliminary analysis of the mitochondrial locus COII showed a similarly high reported interspecific variation and low intraspecific variation as expected (Cook et al. 2005). Finally, the $28 \mathrm{~S}$ ribosomal D2 spacer is a known slow mutating nuclear locus, suited for deeper phylogenetic information (Sallum et al. 2002). We choose three species to use as outgroups: Ae togoi and Ae. hatorii, which belong to the same subgenus as Ae. japonicus, and Ae. vexans, which is more distantly related. All three species are found in the Palearctic and Oriental regions.

Although the combined Bayesian tree is completely resolved into dichotomies, the analysis of parsimony and all individual gene trees return polytomies involving the members of the Ae.japonicus complex and Ae. koreicus. This result likely stems from differences in the way Bayesian and MP approaches manage polytomies (Lewis et al. 2005). Although it is possible that further loci might reduce the level of polytomy, it is equally plausible that the trees reflect a quasi-simultaneous split of the original population into isolated units possibly related to the complex geology and tectonics of Japan and surrounding regions (Taira 2001). A split into northern and southern lines, that independently colonized the Ryukyu archipelago, would explain why the two southern subspecies are more closely related to each other than to any of the other species as well as the seemingly disjunct distribution across the archipelago.

The consistent relationship observed between Ae. koreicus and the subspecies of Ae. japonicus in all molecular markers used, suggests that a new taxonomic construct for the Ae.japonicus complex should be considered, in which the subspecies are raised to species or Ae. koreicus is reclassified as a subspecies. Although all the morphologically differentiating characteristics found in adults and larvae of Ae. koreicus 
and Ae. japonicus s.l. overlap (Tanaka et al. 1979), mating incompatibilities have been demonstrated between Ae. koreicus and Ae.j.japonicus (Miyagi and Lee 1975). Ae. j. japonicus and Ae. koreicus also display important behavioral differences where they are sympatric: in southern Korea Peninsula, Ae. j. japonicus is primarily a forest and rural dwelling mosquito, whereas Ae. koreicus is better adapted to urban environments (Tanaka et al. 1979). Our sampling did not include specimens from mainland China, where other sibling species, subspecies, or even intermediate forms may exist. However, the current evidence of strong genetic isolation among the subspecies of Ae.japonicus and Ae. koreicus, as well as evidence of ecological isolation in sympatry, does fulfill many of the required criteria for species level recognition (i.e., biological, ecological, and evolutionary). Indeed, as summarized in DeQueiroz (2007), “. . . any evidence of lineage separation is sufficient to infer the existence of separate species."

It is perhaps surprising, or just a matter of chance, that the most recent introduction to the United States was Ae.j.japonicus and not Ae. koreicus. There is clear evidence that Ae. japonicus has been introduced multiple times both into the United States (Fonseca et al. 2010) and across the world (Laird et al. 1994, Schaffner et al. 2009); therefore, there is the distinct possibility that Ae. koreicus will also begin spreading across the world. In anticipation of such an event, we developed a DNA-based rapid assay to differentiate Ae. koreicus from some of the species with which it may be confused. Besides Ae. japonicus, in the United States these include Ae. atropalpus and Ae. triseriatus two endemic mosquito species that colonize the same habitats. The assay requires minimal laboratory supplies because the unique extra band in Ae. koreicus specimens can be easily visualized on an agarose gel. The phylogenetic analysis of the Ae. japonicus complex led us to the reexamination of their taxonomic status, as well as the recognition of another potentially invasive species, Ae. koreicus.

\section{Acknowledgments}

We thank all the researchers and mosquito control personnel that provided us with specimens for this research. Without their generosity our work would not have been possible. This material has been reviewed by the Walter Reed Army Institute of Research (WRAIR). There is no objection to its presentation and/or publication. This research was performed under a Memorandum of Understanding between WRAIR and the Smithsonian Institution, with institutional support provided by both organizations. This project has been funded in part by Federal funds from the National Institutes of Health National Institute of Allergy and Infectious Disease, under contract N01-AI25490. This is New Jersey Agricultural Experiment Station publication D-08-0819414-09.

\section{References Cited}

Andreadis, T. G., J. F. Anderson, L. E. Munstermann, R. J. Wolfe, and D. A. Florin. 2001. Discovery, distribution, and abundance of the newly introduced mosquito Ochlerotatus japonicus (Diptera: Culicidae) in Connecticut, USA. J. Med. Entomol. 38: 774-779.

Bevins, S. N. 2007. Establishment and abundance of a recently introduced mosquito species Ochlerotatus japonicus (Diptera: Culicidae) in the southern Appalachians, USA. J. Med. Entomol. 44: 945-952.

Black, W.C.T. 2004. Learning to use Ochlerotatus is just the beginning. J. Am. Mosq. Control Assoc. 20: 215-216.

Cook, S., M. Diallo, A. A. Sall, A. Cooper, and E. C. Holmes. 2005. Mitochondrial markers for molecular identification of Aedes mosquitoes (Diptera: Culicidae) involved in transmission of arboviral disease in West Africa. J. Med. Entomol. 42: 19-28.

de Lamballerie, X., E. Leroy, R. N. Charrel, K. Ttsetsarkin, S. Higgs, and E. A. Gould. 2008. Chikungunya virus adapts to tiger mosquito via evolutionary convergence: a sign of things to come? Virol. J. 5: 33-36.

DeQueiroz, K. 2007. Species concepts and species delimitation. Syst. Biol. 56: 879-886.

Edgecombe, G. D., G.D.F. Wilson, D. J. Colgan, M. R. Gray, and G. Cassis. 2000. Arthropod cladistics: combined analysis of histone H3 and U2 snRNA sequences and morphology. Cladistics 16: 155-203.

Falco, R. C., T. J. Daniels, and M. C. Slameck. 2002. Prevalence and distribution of Ochlerotatus japonicus (Diptera: Culicidae) in two counties in southern New York State. J. Med. Entomol. 39: 920-925.

Fonseca, D. M., D. A. LaPointe, and R. C. Fleischer. 2000. Bottlenecks and multiple introductions: population genetics of the vector of avian malaria in Hawaii. Mol. Ecol. 9: 1803-1814.

Fonseca, D. M., S. Campbell, W. J. Crans, M. Mogi, I. Miyagi, T. Toma, M. Bullians, T. G. Andreadis, R. L. Berry, B. Pagac, M. R. Sardelis, and R. C. Wilkerson. 2001. Aedes (Finlaya) japonicus (Diptera: Culicidae), a newly recognized mosquito in the United States: analyses of genetic variation in the United States and putative source populations. J. Med. Entomol. 38: 135-146.

Fonseca, D. M., A. K. Widdel, M. Hutchinson, S.-E. Spichiger, and L. D. Kramer. 2010. Fine-scale spatial and temporal population genetics of Aedes japonicus, a new US mosquito, reveal multiple introductions. Mol. Ecol. 19(8): 1559-1572.

Foster, W. A., and E. D. Walker. 2002. Mosquitoes (Culicidae), pp. 203-262. In G. Mullen and L. Durden [eds.], Medical and veterinary entomology. Academic, San Diego, CA.

Gratz, N. G. 2004. Critical review of the vector status of Aedes albopictus. Med. Vet. Entomol. 18: 215-227.

Hall, T. 1999. BioEdit computer program, version 7.0.9. (http://www.mbio.ncsu.edu/BioEdit/bioedit.html).

Hughes, T. H., P. M. Irwin, A. Kaufman, H. Sage, B. B. Pagac, Jr., and S. M. Paskewitz. 2008. First records of Aedes japonicus japonicus in Wisconsin. J. Am. Mosq. Control Assoc. 24: 583-584.

Joy, J. E. 2004. Larval mosquitoes in abandoned tire pile sites from West Virginia. J. Am. Mosq. Control Assoc. 20: 12-17.

Kutz, F. W., T. G. Wade, and B. B. Pagac. 2003. A geospatial study of the potential of two exotic species of mosquitoes to impact the epidemiology of West Nile virus in Maryland. J. Am. Mosq. Control Assoc. 19: 190-198.

Laird, M., L. Calder, R. C. Thornton, R. Syme, P. W. Holder, and M. Mogi. 1994. Japanese Aedes albopictus among four mosquito species reaching New Zealand in used tires. J. Am. Mosq. Control Assoc. 10: 14-23. 
Larish, L. B., and H. M. Savage. 2005. Introduction and establishment of Aedes (Finlaya) japonicus japonicus (Theobald) on the island of Hawaii: implications for arbovirus transmission. J. Am. Mosq. Control Assoc. 21: 318-321.

Lewis, P. O., M. T. Holder, and K. E. Holsinger. 2005. Polytomies and Bayesian phylogenetic inference. Syst. Biol. 54: 241-53.

Lounibos, L. P. 2002. Invasions by insect vectors of human disease. Annu. Rev. Entomol. 47: 233-266.

Lu, B. et al. 1997. Fauna Sinica, Insecta. Vol. 8, Diptera: Culicidae 1. Science Press, Beijing, China.

Miyagi, I., and K. W. Lee. 1975. Morphology and biology of Aedes japonicus and Aedes koreicus observed in laboratory experiments. Jpn. J. Sanit. Zool. 25: 300.

Moore, C. G., D. B. Francy, D. A. Eliason, R. E. Bailey, and E. G. Campos. 1990. Aedes albopictus and other container-inhabiting mosquitoes in the United States: results of an eight-city survey. J. Am. Mosq. Control Assoc. 6: 173178.

Morris, J. A., R. L. Lampman, G. Ballmes, J. Funes, J. Halvorsen, and R. J. Novak. 2007. First record of Aedes japonicus japonicus in Illinois: defining its spatial distribution and associated mosquito species. J. Am. Mosq. Control Assoc. 23: 243-251.

Neitzel, D. F., K. A. Johnson, S. Brogren, and M. M. Kemperman. 2009. First collection records of Aedes japonicus in Minnesota. J. Am. Mosq. Control Assoc. 25: $367-369$

Nuin, P. 2006. MrMTgui computer program, version 1.0. (http:// www.genedrift.org/mtgui.php).

Nylander, J.A.A. 2004. MrModeltest computer program, version 2. Evolutionary Biology Centre, Uppsala University, Uppsala, Sweden.

Paupy, C., B. Ollomo, B. Kamgang, S. Moutailler, D. Rousset, M. Demanou, J. P. Herve, E. Leroy, and F. Simard. 2009. Comparative role of Aedes albopictus and Aedes aegypti in the emergence of dengue and chikungunya in Central Africa. Vector Borne Zoonotic Dis: 10.1089/vbz. 2009.0005.

Peyton, E. L., S. R. Campbell, T. M. Candeletti, M. Romanowski, and W. J. Crans. 1999. Aedes (Finlaya) japonicus japonicus (Theobald), a new introduction into the United States. J. Am. Mosq. Control Assoc. 15: 238 241.

Posada, D., and K. A. Crandall. 1998. MODELTEST: testing the model of DNA substitution. Bioinformatics 14: 817818.

Qualls, W. A., and G. R. Mullen. 2006. Larval survey of tire-breeding mosquitoes in Alabama. J. Am. Mosq. Control Assoc. 22: 601-608.

Rambaut, A. 2009. FigTree computer program, version 1.2.2. (http:/ / tree.bio.ed.ac.uk/software/figtree/)

Reinert, J. F. 2000. New classification for the composite genus Aedes (Diptera: Culicidae: Aedini), elevation of subgenus Ochlerotatus to generic rank, reclassification of the other subgenera, and notes on certain subgenera and species. J. Am. Mosq. Control Assoc. 16: 175-188.

Reinert, J. F., R. E. Harbach, and I. J. Kitching. 2004. Phylogeny and classification of Aedini (Diptera: Culicidae) based on morphological characters of all life stages. Zool. J. Linn. Soc. 142: 289-368.

Reinert, J. F., R. E. Harbach, and I. J. Kitching. 2006. Phylogeny and classification of Finlaya and allied taxa (Diptera: Culicidae: Aedini) based on morphological data from all life stages. Zool. J. Linn. Soc. 148: 1-101.

Reinert, J. F., R. E. Harbach, and I. J. Kitching. 2008. Phylogeny and classification of Ochlerotatus and allied taxa
(Diptera: Culicidae: Aedini) based on morphological data from all life stages. Zool. J. Linn. Soc. 153: 29-114.

Ronquist, F., and J. P. Huelsenbeck. 2003. MrBayes 3: Bayesian phylogenetic inference under mixed models. Bioinformatics 19: 1572-1574.

Roppo, M. R., J. L. Lilja, F. A. Maloney, and W. J. Sames. 2004. First occurrence of Ochlerotatus japonicus in the state of Washington. J. Am. Mosq. Control Assoc. 20: 83-84.

Saenz, V. L., L. H. Townsend. R. M. Vanderpool, M. J. Schardein, R. T. Trout, and G. C. Brown. 2006 Ochlerotatus japonicus japonicus in the state of Kentucky. J. Am. Mosq. Cont. Asso. 22: 754-755.

Sallum, M. A., E. S. Bergo, D. C. Flores, and O. P. Forattini. 2002. Systematic studies on Anopheles galvaoi Causey, Deane \& Deane from the subgenus Nysssorhynchus blanchard (Diptera: Culicidae). Mem. Inst. Oswaldo Cruz 97: 1177-1189.

Sardelis, M. R., M. J. Turell, and R. G. Andre. 2002a. Laboratory transmission of La Crosse virus by Ochlerotatus $j$. japonicus (Diptera: Culicidae). J. Med. Entomol. 39: 635639.

Sardelis, M. R., M. J. Turell, and R. G. Andre. 2003. Experimental transmission of St. Louis encephalitis virus by Ochlerotatus j. japonicus. J. Am. Mosq. Control Assoc. 19 159-162.

Sardelis, M. R., D. J. Dohm, B. Pagac, R. G. Andre, and M. J. Turell. 2002b. Experimental transmission of eastern equine encephalitis virus by Ochlerotatus $j$. japonicus (Diptera: Culicidae). J. Med. Entomol. 39: 480-484.

Savage, H. M., and D. Strickman. 2004. The genus and subgenus categories within Culicidae and placement of Ochlerotatus as a subgenus of Aedes. J. Am. Mosq. Control Assoc. 20: 208-214.

Schaffner, F., S. Chouin, and J. Guilloteau. 2003. First record of Ochlerotatus (Finlaya) japonicus japonicus (Theobald, 1901) in metropolitan France. J. Am. Mosq. Control Assoc. 19: 1-5.

Schaffner, F., C. Kaufmann, D. Hegglin, and A. Mathis. 2009. The invasive mosquito Aedes japonicus in Central Europe. Med. Vet. Entomol. 23: 448-451.

Simon, C., F. Frati, A. Beckenbach, B. Crespi, H. Liu, and P. Flook. 1994. Evolution, weighting, and phylogenetic utility of mitochondrial gene sequences and a compilation of conserved polymerase chain reaction primers Ann. Entomol. Soc. Am. 87: 651-701.

Sorenson, M. D., and E. A. Franzosa. 2007. TreeRot computer program, version 3. By M. D. Sorenson and E. A Franzosa. Boston University, Boston, MA.

Swofford, D. L. 2000. PAUP*. Phylogenetic analysis using parsimony (*and other methods) computer program, version 4b10. By D. L. Swofford, Sinauer Associates, Sunderland, MA.

Tabachnick, W. J. 2005. The name game: thoughts on the proposed reclassification of Aedini. Buzz Words-Newl. Fla. Mosq. Control Assoc. 5: 9.

Tabachnick, W. J., and J. R. Powell. 1979. A world-wide survey of genetic variation in the yellow fever mosquito, Aedes aegypti. Genet. Res. 34: 215-229.

Taira, A. 2001. Tectonic evolution of the Japanese Island arc system. Annu. Rev. Earth Planet. Sci. 29: 109-134.

Takashima, I., and L. Rosen. 1989. Horizontal and vertical transmission of Japanese encephalitis virus by Aedes japonicus (Diptera: Culicidae). J. Med. Entomol. 26: 454458.

Tanaka, K., K. Mizusawa, and E. S. Saugstad. 1979. A revision of the adult and larval mosquitoes of Japan (including the Ryukyu archipelago and the Ogasawara islands) and Ko- 
rea (Diptera: Culicidae). Contrib. Am. Entomol. Inst. 16: 1-987.

Thompson, J. D., D. G. Higgins, and T. J. Gibson. 1994. CLUSTAL W: improving the sensitivity of progressive multiple sequence alignment through sequence weighting, position-specific gap penalties and weight matrix choice. Nucleic Acids Res. 22: 4673-4680.

Toma, T., and I. Miyagi. 1981. Notes on the mosquitoes collected at forest areas in the northern part of Okinawajima, Ryukyu Islands, Japan. Jpn. J. Sanit. Zool. 32: 271279.

Toma, T., and I. Miyagi. 1986. The mosquito fauna of the Ryukyu archipelago with identification keys, pupal descriptions and notes on the biology, medical importance and distribution. Mosq. Syst. 18: 1-109.
Turell, M. J., M. R. Sardelis, D. J. Dohm, and M. L. O'Guinn. 2001. Potential North American vectors of West Nile virus. Ann. N Y Acad. Sci. 951: 317-324.

Versteirt, V., F. Schaffner, C. Garros, W. Dekoninck, M. Coosemans, and W. Van Bortel. 2009. Introduction and establishment of the exotic mosquito species Aedes japonicus japonicus (Diptera: Culicidae) in Belgium. J. Med. Entomol. 46: 1464-1467.

Widdel, A. K., L. J. McCuiston, W. J. Crans, L. D. Kramer, and D. M. Fonseca. 2005. Finding needles in the haystack: single copy microsatellite loci for Aedes japonicus (Diptera: Culicidae). Am. J. Trop. Med. Hyg. 73: 744748 .

Received 28 October 2009; accepted 22 February 2010. 